\title{
Status and prospects of light bino-higgsino dark matter in natural SUSY
}

\author{
Murat Abdughani ${ }^{2,3}$, Lei Wu ${ }^{1, a}$, Jin Min Yang ${ }^{2,3}$ \\ ${ }^{1}$ Department of Physics and Institude of Theoretical Physics, Nanjing Normal University, Nanjing, Jiangsu 210023, China \\ 2 CAS Key Laboratory of Theoretical Physics, Institute of Theoretical Physics, Chinese Academy of Sciences, Beijing 100190, China \\ ${ }^{3}$ School of Physical Sciences, University of Chinese Academy of Sciences, Beingjing 100049, China
}

Received: 26 October 2017 / Accepted: 19 December 2017 / Published online: 30 December 2017

(C) The Author(s) 2017. This article is an open access publication

\begin{abstract}
Given the recent progress in dark matter direction detection experiments, we examine a light bino-higgsino dark matter $(\mathrm{DM})$ scenario $\left(M_{1}<100 \mathrm{GeV}\right.$ and $\mu<300$ $\mathrm{GeV}$ ) in natural supersymmetry with the electroweak fine tuning measure $\Delta_{\mathrm{EW}}<30$. By imposing various constraints, we note that: (i) For $\operatorname{sign}\left(\mu / M_{1}\right)=+1$, the parameter space allowed by the DM relic density and collider bounds can almost be excluded by the very recent spin-independent (SI) scattering cross-section limits from the XENON1T (2017) experiment. (ii) For $\operatorname{sign}\left(\mu / M_{1}\right)=$ -1 , the SI limits can be evaded due to the cancelation effects in the $h \tilde{\chi}_{1}^{0} \tilde{\chi}_{1}^{0}$ coupling, while rather stringent constraints come from the PandaX-II (2016) spin-dependent (SD) scattering cross-section limits, which can exclude the higgsino mass $|\mu|$ and the LSP mass $m_{\tilde{\chi}_{1}^{0}}$ up to about 230 and $37 \mathrm{GeV}$, respectively. Furthermore, the surviving parameter space will be fully covered by the projected XENON1T experiment or the future trilepton searches at the HL-LHC.
\end{abstract}

\section{Introduction}

Scrutinizing the mechanism for stabilizing the electroweak scale becomes more impending after the Higgs discovery at the LHC [1,2]. Besides, there is overwhelming evidence for the existence of dark matter from cosmological observations. Identifying the nature of dark matter is one of the challenges in particle physics and cosmology.

The weak scale supersymmetry is widely regarded as one of the most appealing new physics models at the $\mathrm{TeV}$ scale. It can successfully solve the naturalness problem in the Standard Model (SM) and also provide a compelling cold dark matter candidate. Among various supersymmet-

\footnotetext{
a e-mail: leiwu@itp.ac.cn
}

ric models, the natural supersymmetry is a well motivated framework (see for example [3-11]), which usually indicates the presence of light higgsinos in the spectrum [12]. If unification of the gaugino mass parameters is further assumed, the current LHC bound on the gluino $\left(m_{\tilde{g}} \gtrsim\right.$ $2 \mathrm{TeV}$ [13]) would imply correspondingly heavy winos and binos, resulting in a higgsino-like lightest supersymmetric particle (LSP). However, the thermal abundance of light higgsino-like LSP is typically lower than the observed value of the dark matter in the universe, due to the large higgsino-higgsino annihilation rate. These considerations motivate us to explore the phenomenology of neutralino dark matter in natural SUSY by giving up the gaugino mass unification assumption. One of the possibilities is to allow for the light bino in natural SUSY. Such a mixed bino-higgsino neutralino dark matter can solve the abovementioned problems of a pure higgsino LSP without worsening the naturalness in natural SUSY. The studies of bino-higgsino dark matter have also been carried out in [14-33].

In this work, we will confront the light bino-higgsino dark matter scenario in natural SUSY with the recent direct detection data. In particular, we focus on the light dark matter regime $\left(m_{\tilde{\chi}_{1}^{0}}<100 \mathrm{GeV}\right)$ and attempt to address the lower limit of the mass of LSP that saturates the dark matter relic abundance. In natural SUSY, a small $\mu$ parameter leads to a certain bino-higgsino mixing, so that the spin-independent/dependent neutralino LSP-nucleon scattering cross sections can be enhanced. We will utilize the recent XENON1T [34] and PandaX-II [35] limits to examine our parameter space. Since the couplings of the LSP with the SM particles depend on the relative sign $\left(\operatorname{sign}\left(\mu / M_{1}\right)\right)$ between the mass parameters $\mu$ and $M_{1}$, we will include both of $\operatorname{sign}\left(\mu / M_{1}\right)= \pm 1$ in our study and show the impact on the exclusion limits for our scenario. Besides, we explore the potential to probe such a 
scenario by searching for the trilepton events at $14 \mathrm{TeV}$ LHC.

The structure of this paper is organized as follows. In Sect. 2, we will discuss the light bino-higgsino neutralino parameter space in natural SUSY. In Sect. 3, we will perform the parameter scan and discuss our numerical results. Finally, we draw our conclusions in Sect. 4.

\section{Light bino-higgisino neutralino in natural SUSY}

In the MSSM, the minimization of the tree-level Higgs potential leads to the following equation [36]:

$\frac{M_{Z}^{2}}{2}=\frac{m_{H_{d}}^{2}-m_{H_{u}}^{2} \tan ^{2} \beta}{\tan ^{2} \beta-1}-\mu^{2}$,

where $m_{H_{u, d}}^{2}$ denote the soft SUSY breaking masses of the Higgs fields at the weak scale, respectively. It should be noted that the radiative EWSB condition usually imposes a nontrivial relation between the relevant soft mass parameters at the high scale in a UV model, such as mSUGRA. However, the scenario we studied in our work is the so-called low energy phenomenological MSSM, in which a successful EWSB is always assumed and in this case the above mentioned strong correlation between parameters from radiative EWSB condition in UV models is not applicable. Using the electroweak fine tuning measure $\Delta_{\mathrm{EW}}[6]$, one can see that the higgsino mass parameter $\mu$ should be of the order of $\lesssim 300 \mathrm{GeV}$ to satisfy the requirement of $\Delta_{\mathrm{EW}}<30$ [3740]. The light higgsinos have been searched for through chargino pair production in the LEP-2 experiment [41], which indicates $\mu \gtrsim 100 \mathrm{GeV}$. We will use this LEP-2 limit as a lower bound for the higgsino mass. However, the relic abundance of thermally produced pure higgsino LSP falls well below dark matter measurements, unless its mass is in the $\mathrm{TeV}$ range. In order to provide the required relic density, several alternative ways have been proposed, such as the multi-component dark matter on introducing the axion [42]. On the other hand, without fully saturating the relic density (under-abundance), the higgsino-like neutralino dark matter in radiatively driven natural supersymmetry with $\Delta_{\text {EW }}<30$ [43] or the natural mini-landscape model [44] has been confronted with various (in-)direct detections and is also expected to be accessible via the Xenon1T experiment. In our study, we achieve the correct dark matter relic density by allowing the light bino to mix with the higgsinos.

The two neutral higgsinos $\left(\tilde{H}_{u}^{0}\right.$ and $\left.\tilde{H}_{d}^{0}\right)$ and the two neutral gauginos $\left(\tilde{B}\right.$ and $\left.\tilde{W}^{0}\right)$ are combined to form four mass eigenstates called neutralinos. In the gauge-eigenstate basis $\left(\tilde{B}, \tilde{W}^{0}, \tilde{H}_{d}, \tilde{H}_{u}\right)$, the neutralino mass matrix takes the form

$$
M_{\tilde{\chi}^{0}}=\left(\begin{array}{cccc}
M_{1} & 0 & -c_{\beta} s_{W} m_{Z} & s_{\beta} s_{W} m_{Z} \\
0 & M_{2} & c_{\beta} c_{W} m_{Z} & -s_{\beta} s_{W} m_{Z} \\
-c_{\beta} s_{W} m_{Z} & c_{\beta} c_{W} m_{Z} & 0 & -\mu \\
s_{\beta} s_{W} m_{Z} & -s_{\beta} s_{W} m_{Z} & -\mu & 0
\end{array}\right)
$$

where $s_{\beta}=\sin \beta, c_{\beta}=\cos \beta, s_{W}=\sin \theta_{W}, c_{W}=\cos \theta_{W}$, $M_{1}$ and $M_{2}$ are the soft-breaking mass parameters for bino and wino, respectively. $M_{\tilde{\chi}^{0}}$ can be diagonalized by a $4 \times 4$ unitary matrix $N$. In the limit of $M_{1}<\mu \ll M_{2}$, the lightest neutralino is bino-like (with some higgsino mixture), while the second and third neutralinos are higgsino-like. The LSP can interact with nuclei via exchange of squarks and Higgs bosons (spin-independent) and via exchange of $Z$ boson and squarks (spin-dependent). Given the strong LHC bounds on the squarks and non-SM Higgs bosons, one can neglect their contributions to the scattering cross section. Then the couplings of the LSP with the Higgs boson can be written

$C_{h \tilde{\chi}_{1}^{0} \tilde{\chi}_{1}^{0}} \approx-\sqrt{2} g_{1} N_{11}^{2} \frac{M_{Z} s_{W}}{\mu} \frac{M_{1} / \mu+\sin 2 \beta}{1-\left(M_{1} / \mu\right)^{2}}$,

where $N_{11}$ denotes the bino component of the lightest neutralino mass eigenstate. It can be seen that the SI scattering cross section depends on the relative sign of $M_{1}$ and $\mu$. When $\operatorname{sign}\left(M_{1} / \mu\right)<0$, the coupling $C_{h \tilde{\chi}_{1}^{0} \tilde{\chi}_{1}^{0}}$ can be suppressed and even vanish if $M_{1} / \mu=-\sin 2 \beta$ so that the strong LUX SI limits can be escaped. For the SD scattering cross section, it should be noted that the coupling $Z \tilde{\chi}_{1}^{0} \tilde{\chi}_{1}^{0}$ can appear via the higgsino component in the LSP. The pure bino/wino LSP will not have interactions with the $Z$ boson, while the pure higgsino LSP can only have the non-zero coupling $Z \tilde{\chi}_{1}^{0} \tilde{\chi}_{2}^{0}$. Another blind spot in SD scattering may occur in the limit of $\tan \beta=1$, where the left-right parity is restored and the parity-violating $Z$ coupling will vanish [16]. However, a low value of $\tan \beta$ is disfavored by the observed Higgs mass in the MSSM.

\section{Parameter scan and numerical results}

In our numerical calculations, we vary the relevant parameters in the ranges of

$$
\begin{aligned}
100 \mathrm{GeV} & \leq|\mu| \leq 300 \mathrm{GeV}, 30 \mathrm{GeV} \\
& \leq\left|M_{1}\right| \leq 100 \mathrm{GeV}, 10 \leq \tan \beta \leq 50 .
\end{aligned}
$$

We scan the values of $M_{1}$ up to $100 \mathrm{GeV}$ since we are interested in light DM region and attempt to address the lower limit of the LSP mass. For higher upper values of $\mu$ and $M_{1}$, a heavy mixed higgsino-bino LSP may also produce the right DM relic abundance [20], while the result for lower bound of LSP mass obtained in the following calculation will not change. The stop and gluino can contribute to the naturalness at loop level, which are expected to be $m_{\tilde{t}_{1}} \lesssim 2.5 \mathrm{TeV}$ 

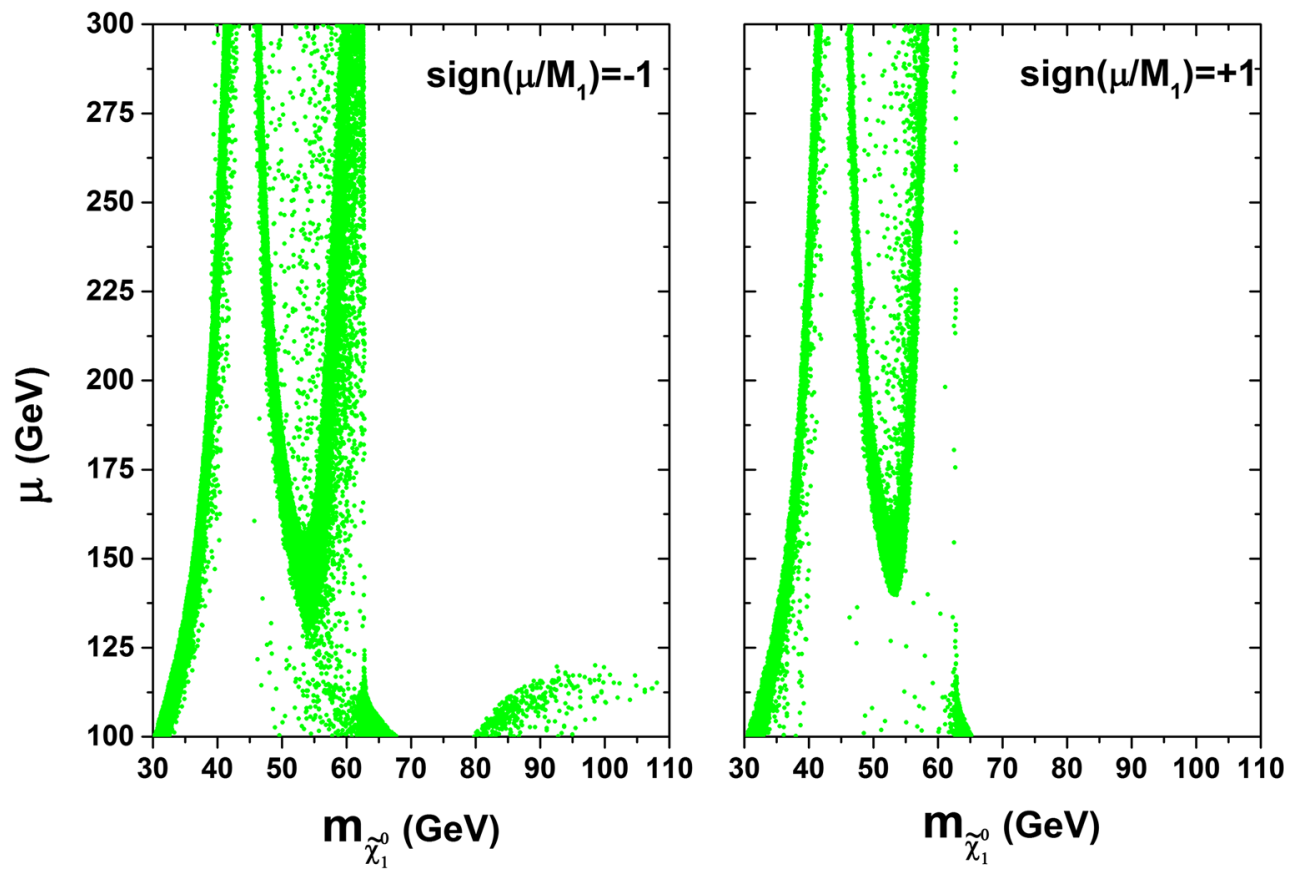

Fig. 1 Scattering plot of samples satisfying the dark matter relic density

and $m_{\tilde{g}} \lesssim 3-4 \mathrm{TeV}$ for $\Delta_{\mathrm{EW}}<30$ [37,45]. By recasting the LHC Run- 2 with $\sim 15 \mathrm{fb}^{-1}$ of data, it is found that the lower bounds of stop mass and gluino mass are about 800 GeV [46-51] and $1.5 \mathrm{TeV}$ [52] in natural SUSY, respectively. Given the irrelevance of the third generation parameters for our neutralino dark matter, we fix the third generation squark soft masses as $M_{\tilde{Q}_{3 L}}=3 \mathrm{TeV}, M_{\tilde{t}_{3 R}}=M_{\tilde{b}_{3 R}}=1 \mathrm{TeV}$ and vary the stop trilinear parameters in the range $\left|A_{t}\right|<2 \mathrm{TeV}$ for simplicity. The physical stop mass $m_{\tilde{t}_{1}}$ has to be less than $2.5 \mathrm{TeV}$ to satisfy $\Delta_{\mathrm{EW}}<30$. We also require that each sample can guarantee the correct Higgs mass and the vacuum stability $[53,54]$. For the first two generations, the squark and all slepton soft masses are assumed to be $3 \mathrm{TeV}$. Other trilinear parameters are fixed as $A_{f}=0$. We also decouple the wino and gluino by setting $M_{2,3}=2 \mathrm{TeV}$. We impose the following constraints in our scan:

(1) The light CP-even Higgs boson masses of our samples should be within the range of $122-128 \mathrm{GeV}$. The package SuSpect [55] is used to calculate the Higgs mass.

(2) The samples have to be consistent with the Higgs data from LEP, Tevatron and LHC. We use the packages HiggsBounds-4.2.1 [56,57] and HiggsSignals-1.4.0 [58] to implement the constraints.

(3) The relic density of neutralino dark matter $\Omega_{\tilde{\chi}} h^{2}$ is computed by MicrOMEGAs 4.3.2 [59]. Including 10\% theoretical uncertainty, we require our samples to satisfy the observed value $0.1186 \pm 0.0020[60]$ within $2 \sigma$ range.
(4) If $m_{\tilde{\chi}_{1}^{0}}<m_{h} / 2$, the SM Higgs boson can decay to $\tilde{\chi}_{1}^{0} \tilde{\chi}_{1}^{0}$ invisibly. We require the branching ratio $\mathrm{Br}(h \rightarrow$ $\left.\tilde{\chi}_{1}^{0} \tilde{\chi}_{1}^{0}\right)<24 \%$, which has recently been given by the CMS collaboration at $95 \%$ C.L. [61].

(5) The invisible width of the $Z$ boson is required to be less than $0.5 \mathrm{MeV}$ to satisfy the LEP limit.

(6) The LEP searches for $\tilde{\chi}_{1}^{0} \tilde{\chi}_{2,3}^{0}$ associated production gives an upper limit, $\sigma\left(e^{+} e^{-} \rightarrow \tilde{\chi}_{1}^{0} \tilde{\chi}_{2,3}^{0} \times \operatorname{Br}\left(\tilde{\chi}_{2,3}^{0} \rightarrow\right.\right.$ $\left.\tilde{\chi}_{1}^{0} Z^{*}\right)<100 \mathrm{fb}$.

In Fig. 1, we show the samples satisfying the dark matter relic density for $\operatorname{sign}(\mu)= \pm 1$. Since a bino-like LSP has rather small couplings with the SM particles, a certain portion of higgsino components is required to meet the observed relic density. Otherwise, the universe will be overclosed. Therefore, except for the two resonance regions $m_{\tilde{\chi}_{1}^{0}} \simeq m_{Z} / 2$ and $m_{h} / 2$, the higgsino mass parameter $\mu$ is expected to be as low as possible in our scan ranges. It should be noted that the difference of $\operatorname{sign}\left(\mu / M_{1}\right)= \pm 1$ in calculating the relic abundance mainly happens around and after the Higgs resonance region, in which more samples are allowed for $\operatorname{sign}\left(\mu / M_{1}\right)=-1$. This is because the negative sign of $\mu / M_{1}$ can reduce the coupling of the LSP with the Higgs boson and the suppress the enhanced annihilation cross section of $\tilde{\chi}_{1}^{0} \tilde{\chi}_{1}^{0}$ by the Higgs resonant effect. When $m_{\tilde{\chi}_{1}^{0}}>m_{h} / 2$, the LSP for $\operatorname{sign}\left(\mu / M_{1}\right)= \pm 1$ is still binolike so that the relic density easily exceeds the observed value. But if $M_{1}$ is close to $\mu$, the LSP for $\operatorname{sign}\left(\mu / M_{1}\right)=-1$ can have sizable higgsino components, which allows samples in 


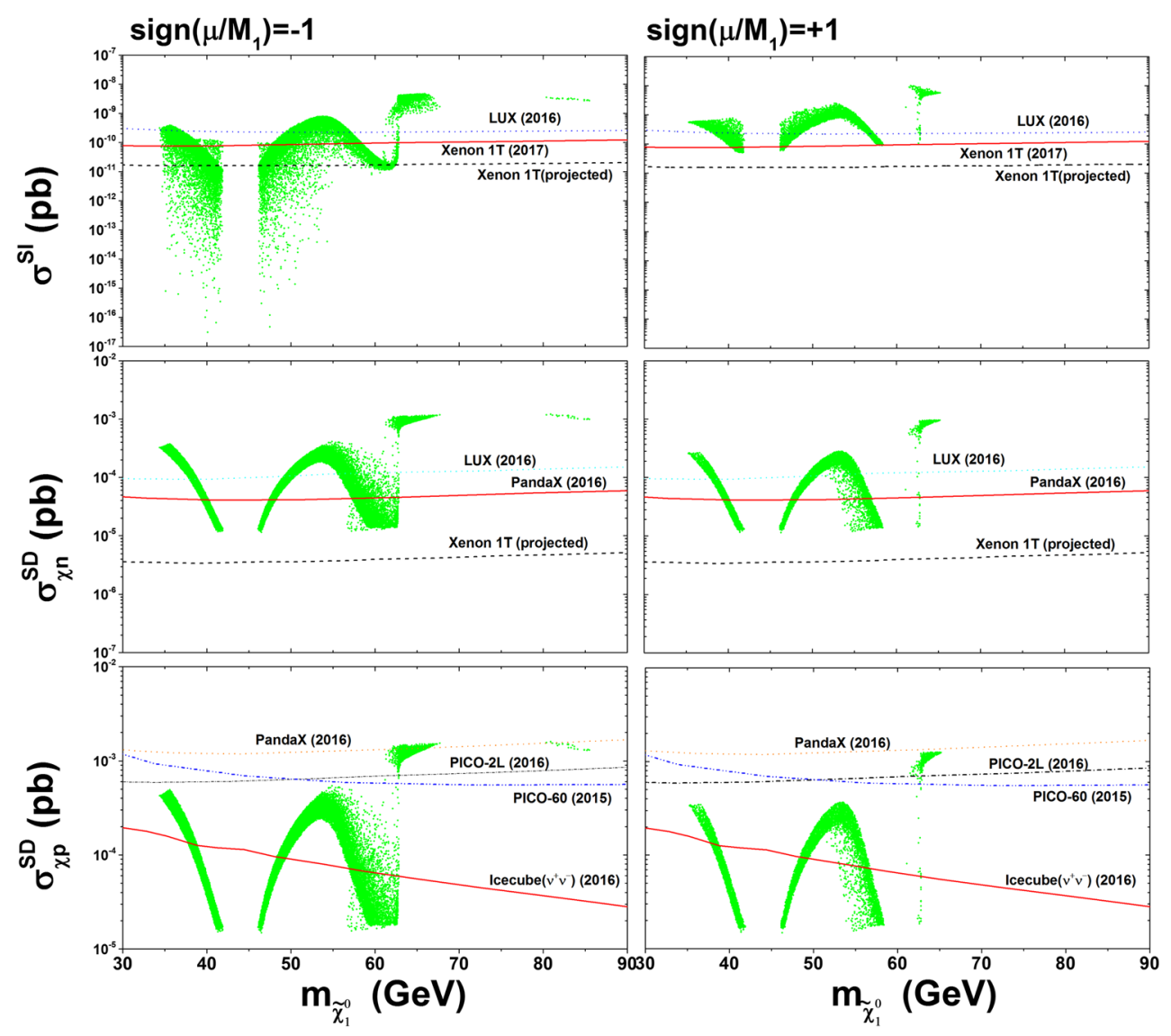

Fig. 2 Spin-independent/dependent neutralino LSP-nucleon scattering cross sections. All samples satisfying the constraints (1-6). The observed $90 \%$ C.L. upper limits from Xenon1T (2017) [34], PandaX (2016) [35], LUX (2016) [62,63], PICO-2L (2016) [64], PICO-60

the lower right corner on the left panel of Fig. 1. However, such a region will be excluded by the dark matter direct detections as shown in the following.

In Fig. 2, we present the spin-independent/dependent neutralino LSP-nucleon scattering cross sections, which are calculated by using MicrOMEGAs 4.3.2 [59]. All samples satisfying the constraints (1-6). The neutron and proton form factors are taken as $f_{d}^{p} \approx 0.132$ and $f_{d}^{n} \approx 0.140$. It can be seen that the very recent SI cross-section limits from XENON1T experiment can almost exclude the whole parameter space of $\operatorname{sign}\left(\mu / M_{1}\right)=+1$. While for $\operatorname{sign}\left(\mu / M_{1}\right)=$ -1 , a large portion of our samples can escape the SI limits since the $h \tilde{\chi}_{1}^{0} \tilde{\chi}_{1}^{0}$ coupling is suppressed by the cancellation effect in Eq. (3).

On the other hand, the SD cross section is largely determined by $Z$-boson exchange and is sensitive to the higgsino asymmetry, $\sigma_{S D} \propto\left|N_{13}^{2}-N_{14}^{2}\right|^{2}$. The relic density constraint requires a large higgsino asymmetry so that the SD cross section is enhanced. Therefore, a strong bound on such a scenario comes from the PandaX-II (2016) SD neutralino
(2015) [65], IceCube (2016) [66] and the projected XENON1T sensitivity limits [67] are plotted. For indirect limits, we assume that LSP annihilates exclusively to some specific final state, with a canonical thermal annihilation cross section $\langle\sigma v\rangle_{0}=3 \times 10^{-26} \mathrm{~cm}^{3} \mathrm{~s}^{-1}$

LSP-neutron scattering cross-section limits, which can rule out about $70 \%$ of our samples and exclude the higgsino mass $|\mu|$ and the LSP mass $m_{\tilde{\chi}_{1}^{0}}$ up to about 230 and $37 \mathrm{GeV}$, respectively. Such lower limits will not changed even if we extend the scan ranges of $M_{1}$ and $\mu$ to larger values. The current SD neutralino LSP-proton limits from PandaX and PICO are still weak. Both of $\operatorname{sign}(\mu)= \pm 1$ scenarios can be completely covered by the projected XENON1T experiment in the future.

Besides the direct detections, the neutralino annihilation in the Sun to neutrinos can also be enhanced by the higgsino component in the LSP. The null results from the neutrino telescopes, such as IceCube, have produced a strong bound on the SD neutralino LSP-proton scattering cross sections and has excluded a sizable portion of the parameter space for $\operatorname{sign}(\mu)=-1$. Next, we discuss the LHC potential of probing the current parameter space of our scenario allowed by the constraints (1)-(6) and the above direct/indirect detections. 

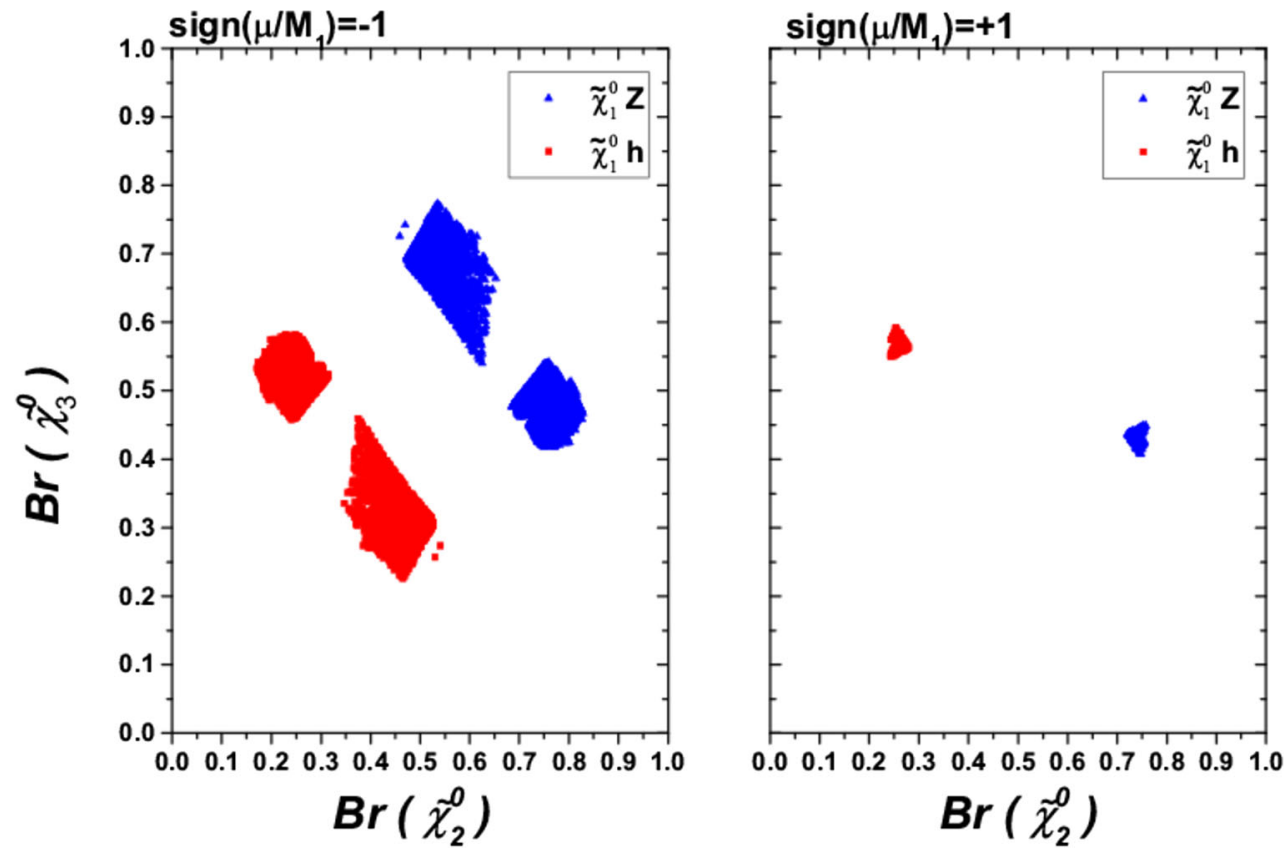

Fig. 3 Scatter plots of the samples allowed by the constraints (1-6) and by the XENON1T (2017) and PandaX (2016), showing $\tilde{\chi}_{2}^{0}$ and $\tilde{\chi}_{3}^{0}$ decay branching ratios

Table 1 Recast LHC-8 TeV analyses with $20.3 \mathrm{fb}^{-1}$ of data and corresponding signals in our scenario

\begin{tabular}{ll}
\hline Final states & Source of signal in our scenario \\
\hline 3lepton $+\mathbb{E}_{T}[77]$ & $p p \rightarrow \tilde{\chi}_{1}^{ \pm}\left(\rightarrow W^{ \pm} \tilde{\chi}_{1}^{0}\right) \tilde{\chi}_{2,3}^{0}\left(\rightarrow Z \tilde{\chi}_{1}^{0}\right)$ \\
1lepton $+h+\mathbb{E}_{T}[78]$ & $p p \rightarrow \tilde{\chi}_{1}^{ \pm}\left(\rightarrow W^{ \pm} \tilde{\chi}_{1}^{0}\right) \tilde{\chi}_{2,3}^{0}\left(\rightarrow h \tilde{\chi}_{1}^{0}\right)$ \\
$\ell^{+} \ell^{-}+\mathbb{E}_{T}[79]$ & $p p \rightarrow \tilde{\chi}_{1}^{+} \tilde{\chi}_{1}^{-}$ \\
\hline
\end{tabular}

In Fig. 3, we plot the decay branching ratios of $\tilde{\chi}_{2}^{0}$ and $\tilde{\chi}_{3}^{0}$. For $\operatorname{sign}(\mu)=-1$, we can see that the neutralinos $\tilde{\chi}_{2,3}^{0}$ mainly decay to $\tilde{\chi}_{1}^{0} Z$. When $\operatorname{Br}\left(\tilde{\chi}_{2}^{0} \rightarrow \tilde{\chi}_{1}^{0} Z\right)$ increases, $\operatorname{Br}\left(\tilde{\chi}_{3}^{0} \rightarrow \tilde{\chi}_{1}^{0} Z\right)$ decreases because of the Goldstone theorem [25]. A similar correlation can be seen in the decay channel $\tilde{\chi}_{2,3}^{0} \rightarrow \tilde{\chi}_{1}^{0} h$. But for $\operatorname{sign}(\mu)=+1$, the neutralino $\tilde{\chi}_{2}^{0}$ still dominantly decay to $\tilde{\chi}_{1}^{0} Z$, while the neutralino $\tilde{\chi}_{3}^{0}$ preferably decay to $\tilde{\chi}_{1}^{0} h$. This indicates that the samples with negative sign of $\mu / M_{1}$ will produce more trilepton events through the process $p p \rightarrow \tilde{\chi}_{2,3}^{0}\left(\rightarrow Z \tilde{\chi}_{1}^{0}\right) \tilde{\chi}_{1}^{ \pm}\left(\rightarrow W^{ \pm} \tilde{\chi}_{1}^{0}\right)$ than those with positive sign of $\mu / M_{1}$, and can be more easily excluded by the null results of searching for electroweakinos at the LHC.

Given the above decay modes, we first recast the LHC searches for the electroweakinos listed in Table 1 with CheckMATE2 [68-70]. We generate the parton level signal events by MadGraph5_aMC @NLO [71] and per- form the shower and hadronization procedure by Pythia8.2 [72]. The fast detector simulation are carried out with the tuned Delphes [73]. We implement the jet clustering by FastJet [74] with the anti- $k_{t}$ algorithm [75]. We use Prospino2 [76] to calculate the QCD corrected cross sections of the electroweakino pair productions at the LHC. Then we estimate the exclusion limit by evaluating the ratio $r=\max \left(N_{S, i} / S_{o b s, i}^{95 \%}\right)$, where $N_{S, i}$ is the event number of signal for $i$ th signal region and $S_{o b s, i}^{95 \%}$ is the corresponding $95 \%$ C.L. observed upper limit. A sample is excluded at $95 \%$ C.L. if $r>1$. After checking all surviving samples, we find that the LHC data in Table 1 cannot further exclude the parameter space because of the strong direct detection bound on higgsino mass parameter $\mu>230 \mathrm{GeV}$.

In Fig. 4, we show the prospect of testing our surviving samples through searching for electroweakino pair production in the trilepton final states at $14 \mathrm{TeV}$ LHC with the luminosity $\mathcal{L}=3000 \mathrm{fb}^{-1}$. Such an analysis [80] has been implemented in CheckMATE package. In order to reduce the Monte Carlo fluctuations, we generate 200,000 events for each signal point. In Fig. 4, we can see that all red triangles allowed by the constraints (1)-(6) and the XENON1T (2017) and PandaX (2016) experiments can be excluded by the HL-LHC at $95 \%$ C.L. Therefore, we conclude that our light bino-higgsino neutralino dark matter scenario will be fully tested by either future XENON1T or HL-LHC experiments. 


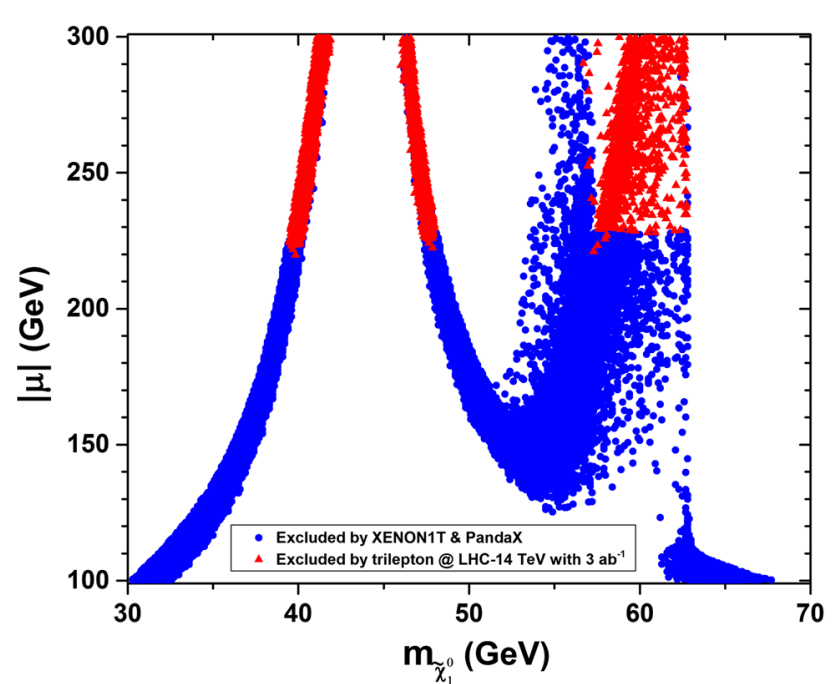

Fig. 4 Scatter plots of the samples allowed by the constraints (1-6) on the plane of $|\mu|$ versus $m_{\tilde{\chi}_{1}^{0}}$. The blue bullets are excluded by the XENON1T (2017) and PandaX (2016). The red triangle are expected to be excluded by the trilepton searches at 95\% C.L. at the HL-LHC

\section{Conclusion}

In this work, we examined light bino-higgsino neutralino dark matter in natural SUSY by imposing various constraints from the LEP, dark matter and LHC experiments. We found that the relative sign between the mass parameters $\mu$ and $M_{1}$ can significantly affect the dark matter and LHC phenomenology of our scenario. For $\operatorname{sign}\left(\mu / M_{1}\right)=+1$, the very recent SI limits from the Xenon1T (2017) experiment can almost exclude the whole parameter space allowed by the relic density and collider bounds. But for $\operatorname{sign}\left(\mu / M_{1}\right)=-1$, the SI limits can be avoided due to the cancellation effects in $h \tilde{\chi}_{1}^{0} \tilde{\chi}_{1}^{0}$ coupling. In this case, a strong bound comes from the PandaX-II (2016) SD neutralino LSP-neutron scattering cross-section limits, which can exclude the higgsino mass $|\mu|$ and the LSP mass $m_{\tilde{\chi}_{1}^{0}}$ up to about 230 and $37 \mathrm{GeV}$, respectively. Furthermore, the surviving parameter space will be fully covered by the projected XENON1T experiment or the future trilepton searches at $14 \mathrm{TeV}$ LHC with the luminosity $\mathcal{L}=3000 \mathrm{fb}^{-1}$.

Acknowledgements We thank G. H. Duan and Yang Zhang for helpful discussions. This work is supported by the National Natural Science Foundation of China (NNSFC) under Grant nos. 11705093 and 11675242, by the CAS Center for Excellence in Particle Physics (CCEPP) and by the CAS Key Research Program of Frontier Sciences.

Open Access This article is distributed under the terms of the Creative Commons Attribution 4.0 International License (http://creativecomm ons.org/licenses/by/4.0/), which permits unrestricted use, distribution, and reproduction in any medium, provided you give appropriate credit to the original author(s) and the source, provide a link to the Creative Commons license, and indicate if changes were made.

Funded by SCOAP ${ }^{3}$.

\section{References}

1. G. Aad et al. [ATLAS Collaboration], Phys. Lett. B 716, 1 (2012). https://doi.org/10.1016/j.physletb.2012.08.020. arXiv:1207.7214 [hep-ex]

2. S. Chatrchyan et al. [CMS Collaboration], Phys. Lett. B 716, 30 (2012). https://doi.org/10.1016/j.physletb.2012.08.021. arXiv:1207.7235 [hep-ex]

3. C. Brust, A. Katz, S. Lawrence, R. Sundrum, JHEP 1203, 103 (2012)

4. M. Papucci, J.T. Ruderman, A. Weiler, JHEP 1209, 035 (2012)

5. L.J. Hall, D. Pinner, J.T. Ruderman, JHEP 1204, 131 (2012)

6. H. Baer, V. Barger, P. Huang, A. Mustafayev, X. Tata, Phys. Rev. Lett. 109, 161802 (2012). arXiv:1207.3343 [hep-ph]

7. J. Cao, C. Han, L. Wu, J.M. Yang, Y. Zhang, JHEP 1211, 039 (2012). https://doi.org/10.1007/JHEP11(2012)039. arXiv:1206.3865 [hep-ph]

8. L. Calibbi, T. Li, A. Mustafayev, S. Raza, Phys. Rev. D 93(11), 115018 (2016). https://doi.org/10.1103/PhysRevD.93. 115018. arXiv:1603.06720 [hep-ph]

9. F. Wang, J.M. Yang, Y. Zhang, JHEP 1604, 177 (2016). https://doi. org/10.1007/JHEP04(2016)177. arXiv:1602.01699 [hep-ph]

10. L. Wu, arXiv:1705.02534 [hep-ph]

11. G .H. Duan, K i Hikasa, L. Wu, J.M. Yang, M. Zhang, JHEP 1703, 091 (2017). https://doi.org/10.1007/JHEP03(2017)091. arXiv:1611.05211 [hep-ph]

12. R. Barbieri, G.F. Giudice, Nucl. Phys. B 306, 63 (1988)

13. The ATLAS collaboration, ATLAS-CONF-2015-067

14. M. Drees, M.M. Nojiri, Phys. Rev. D 47, 376 (1993). https://doi. org/10.1103/PhysRevD.47.376. arXiv:hep-ph/9207234

15. I. Gogoladze, R. Khalid, Y. Mimura, Q. Shafi, Phys. Rev. D 83, 095007 (2011). https://doi.org/10.1103/PhysRevD.83.095007. arXiv:1012.1613 [hep-ph]

16. C. Cheung, L.J. Hall, D. Pinner, J.T. Ruderman, JHEP 1305, 100 (2013). https://doi.org/10.1007/JHEP05(2013)100. arXiv:1211.4873 [hep-ph]

17. B. Dutta, T. Kamon, N. Kolev, K. Sinha, K. Wang, S. Wu, Phys. Rev. D 87(9), 095007 (2013). https://doi.org/10.1103/PhysRevD. 87.095007. arXiv:1302.3231 [hep-ph]

18. G .B. langer, G. Drieu La Rochelle, B. Dumont, R .M. Godbole, S. Kraml, S. Kulkarni, Phys. Lett. B 726, 773 (2013). https://doi.org/ 10.1016/j.physletb.2013.09.059. arXiv:1308.3735 [hep-ph]

19. T.T. Yanagida, N. Yokozaki, JHEP 1311, 020 (2013). https://doi. org/10.1007/JHEP11(2013)020. arXiv:1308.0536 [hep-ph]

20. H. Baer, V. Barger, P. Huang, D. Mickelson, M. Padeffke-Kirkland, X. Tata, Phys. Rev. D 91(7), 075005 (2015). https://doi.org/10. 1103/PhysRevD.91.075005. arXiv:1501.06357 [hep-ph]

21. C. Han, arXiv: 1409.7000 [hep-ph]

22. A. Kobakhidze, M. Talia, L. Wu, Phys. Rev. D 95(5), 055023 (2017). https://doi.org/10.1103/PhysRevD.95.055023. arXiv:1608.03641 [hep-ph]

23. M. Badziak, M. Olechowski, P. Szczerbiak, Phys. Lett. B 770, 226 (2017). https://doi.org/10.1016/j.physletb.2017.04.059. arXiv:1701.05869 [hep-ph]

24. T. Han, F. Kling, S. Su, Y. Wu, JHEP 1702, 057 (2017). https://doi. org/10.1007/JHEP02(2017)057. arXiv:1612.02387 [hep-ph]

25. T. Han, Z. Liu, S. Su, JHEP 1408, 093 (2014). https://doi.org/10. 1007/JHEP08(2014)093. arXiv:1406.1181 [hep-ph]

26. L. Calibbi, J.M. Lindert, T. Ota, Y. Takanishi, JHEP 1411, 106 (2014). https://doi.org/10.1007/JHEP11(2014)106. arXiv:1410.5730 [hep-ph]

27. J. Kawamura, Y. Omura, arXiv:1703.10379 [hep-ph]

28. M. van Beekveld, W. Beenakker, S. Caron, R. Peeters, R. Ruiz de Austri, arXiv:1612.06333 [hep-ph] 
29. M. van Beekveld, W. Beenakker, S. Caron, R. Ruiz de Austri, JHEP 1604, 154 (2016). https://doi.org/10.1007/JHEP04(2016)154. arXiv:1602.00590 [hep-ph]

30. A. Achterberg, S. Amoroso, S. Caron, L. Hendriks, R. Ruiz de Austri, C. Weniger, JCAP 1508(08), 006 (2015). https://doi.org/ 10.1088/1475-7516/2015/08/006. arXiv:1502.05703 [hep-ph]

31. C. Han, K i Hikasa, L. Wu, J .M. Yang, Y. Zhang, Phys. Lett. B 769, 470 (2017). https://doi.org/10.1016/j.physletb.2017.04.026. arXiv:1612.02296 [hep-ph]

32. P. Athron et al. [GAMBIT Collaboration], arXiv:1705.07935 [hep$\mathrm{ph}]$;

33. P. Athron et al. [GAMBIT Collaboration], arXiv:1705.07917 [hep$\mathrm{ph}$;

34. E. Aprile et al. [XENON Collaboration], arXiv:1705.06655 [astroph.CO]

35. C. Fu et al. [PandaX-II Collaboration], Phys. Rev. Lett. 118(7), 071301 (2017). https://doi.org/10.1103/PhysRevLett.118.071301. arXiv:1611.06553 [hep-ex]

36. R. Arnowitt, P. Nath, Phys. Rev. D 46, 3981 (1992)

37. H. Baer, V. Barger, P. Huang, D. Mickelson, A. Mustafayev, X. Tata, Phys. Rev. D 87(11), 115028 (2013). arXiv:1212.2655 [hep-ph]

38. G.G. Ross, K. Schmidt-Hoberg, F. Staub, JHEP 1703, 021 (2017). https://doi.org/10.1007/JHEP03(2017)021. arXiv:1701.03480 [hep-ph]

39. G.G. Ross, K. Schmidt-Hoberg, F. Staub, Phys. Lett. B 759, 110 (2016). https://doi.org/10.1016/j.physletb.2016.05.053. arXiv:1603.09347 [hep-ph]

40. C. Han, A. Kobakhidze, N. Liu, A. Saavedra, L. Wu, J.M. Yang, JHEP 1402, 049 (2014). https://doi.org/10.1007/ JHEP02(2014)049. arXiv:1310.4274 [hep-ph]

41. LEP2 SUSY Working Group, LEPSUSYWG/01-03.1 (2001)

42. H. Baer, A. Lessa, S. Rajagopalan, W. Sreethawong, JCAP 1106, 031 (2011). https://doi.org/10.1088/1475-7516/2011/06/ 031. arXiv:1103.5413 [hep-ph]

43. H. Baer, V. Barger, D. Mickelson, Phys. Lett. B 726, 330 (2013). https://doi.org/10.1016/j.physletb.2013.08.060. arXiv:1303.3816 [hep-ph]

44. H. Baer, V. Barger, M. Savoy, H. Serce, X. Tata, JHEP 1706, 101 (2017). https://doi.org/10.1007/JHEP06(2017)101. arXiv:1705.01578 [hep-ph]

45. H. Baer, V. Barger, P. Huang, X. Tata, JHEP 1205, 109 (2012)

46. C. Han, K i Hikasa, L. Wu, J .M. Yang, Y. Zhang, JHEP 1310, 216 (2013). https://doi.org/10.1007/JHEP10(2013)216. arXiv:1308.5307 [hep-ph]

47. K. Kowalska, E.M. Sessolo, Phys. Rev. D 88(7), 075001 (2013). arXiv: 1307.5790 [hep-ph]

48. A. Kobakhidze, N. Liu, L. Wu, J.M. Yang, M. Zhang, Phys. Lett. B 755, 76 (2016). https://doi.org/10.1016/j.physletb.2016.02.003. arXiv:1511.02371 [hep-ph]

49. J.S. Kim, K. Rolbiecki, R. Ruiz, J. Tattersall, T. Weber, Phys. Rev. D 94(9), 095013 (2016). https://doi.org/10.1103/PhysRevD. 94.095013. arXiv: 1606.06738 [hep-ph]

50. C. Han, J. Ren, L. Wu, J.M. Yang, M. Zhang, Eur. Phys. J. C 77(2), 93 (2017). https://doi.org/10.1140/epjc/ s10052-017-4662-7. arXiv:1609.02361 [hep-ph]

51. H. Baer, V. Barger, N. Nagata, M. Savoy, Phys. Rev. D 95(5), 055012 (2017). https://doi.org/10.1103/PhysRevD.95. 055012. arXiv:1611.08511 [hep-ph]

52. M.R. Buckley, D. Feld, S. Macaluso, A. Monteux, D. Shih, arXiv:1610.08059 [hep-ph]
53. D. Chowdhury, R.M. Godbole, K.A. Mohan, S.K. Vempati, JHEP 1402, 110 (2014). https://doi.org/10.1007/JHEP02(2014)110. arXiv:1310.1932 [hep-ph]

54. N. Blinov, D.E. Morrissey, JHEP 1403, 106 (2014). https://doi.org/ 10.1007/JHEP03(2014)106. arXiv:1310.4174 [hep-ph]

55. A. Djouadi, J.L. Kneur, G. Moultaka, Comput. Phys. Commun. 176, 426 (2007). https://doi.org/10.1016/j.cpc.2006.11.009. arXiv:hep-ph/0211331

56. P. Bechtle et al., Comput. Phys. Commun. 182, 2605 (2011)

57. P. Bechtle et al., Comput. Phys. Commun. 181, 138 (2010)

58. P. Bechtle et al., Eur. Phys. J. C 74, 2711 (2014)

59. G. Belanger et al., Comput. Phys. Commun. 182, 842 (2011)

60. P.A.R. Ade et al. [Planck Collaboration], arXiv:1303.5076 [astroph.CO]

61. V. Khachatryan et al. [CMS Collaboration]. JHEP 1702, 135 (2017). https://doi.org/10.1007/JHEP02(2017)135. arXiv: 1610.09218 [hep-ex]

62. D.S. Akerib et al. (LUX Collaboration), Phys. Rev. Lett. 118, 021303 (2017). arXiv:1608.07648 [astro-ph.CO]

63. D.S. Akerib et al. (LUX Collaboration), Phys. Rev. Lett. 116, 161302 (2016). arXiv:1602.03489 [hep-ex]

64. C. Amole et al. [PICO Collaboration], Phys. Rev. D 93(6), 061101 (2016). https://doi.org/10.1103/PhysRevD.93.061101. arXiv:1601.03729 [astro-ph.CO]

65. C. Amole et al. [PICO Collaboration], Phys. Rev. D 93(5), 052014 (2016). https://doi.org/10.1103/PhysRevD.93.052014. arXiv: 1510.07754 [hep-ex]

66. M.G. Aartsen et al. [IceCube Collaboration], JCAP 1604(04), 022 (2016). https://doi.org/10.1088/1475-7516/2016/04/022. arXiv:1601.00653 [hep-ph]

67. E. Aprile et al. [XENON Collaboration], JCAP 1604(04), 027 (2016). https://doi.org/10.1088/1475-7516/2016/04/027. arXiv:1512.07501 [physics.ins-det]

68. M. Drees et al., Comput. Phys. Commun. 187, 227 (2014)

69. J.S. Kim et al., arXiv:1503.01123 [hep-ph]

70. D. Dercks, N. Desai, J.S. Kim, K. Rolbiecki, J. Tattersall, T. Weber, arXiv:1611.09856 [hep-ph]

71. J. Alwall et al., JHEP 1407, 079 (2014)

72. T. Sjóstrand et al., Comput. Phys. Commun. 191, 159 (2015). https://doi.org/10.1016/j.cpc.2015.01.024. arXiv:1410.3012 [hep$\mathrm{ph}]$

73. J. de Favereau et al., arXiv:1307.6346 [hep-ex]

74. M. Cacciari, G.P. Salam, G. Soyez, Eur. Phys. J. C 72, 1896 (2012). arXiv:1111.6097 [hep-ph]

75. M. Cacciari, G.P. Salam, G. Soyez, JHEP 0804, 063 (2008)

76. W. Beenakker, M. Klasen, M. Kramer, T. Plehn, M. Spira, P.M. Zerwas, Phys. Rev. Lett. 83, 3780 (1999). https://doi.org/ 10.1103/PhysRevLett.83.3780. arXiv:hep-ph/9906298. (Erratum: [Phys. Rev. Lett. 100, 029901 (2008)]. https://doi.org/10.1103/ PhysRevLett.100.029901)

77. G. Aad et al. [ATLAS Collaboration], JHEP 1404, 169 (2014). https://doi.org/10.1007/JHEP04(2014)169. arXiv:1402.7029 [hep-ex]

78. G. Aad et al. [ATLAS Collaboration], Eur. Phys. J. C 75(5), 208 (2015). https://doi.org/10.1140/epjc/s10052-015-3408-7. arXiv: 1501.07110 [hep-ex]

79. [ATLAS Collaboration], ATLAS-CONF-2013-049

80. [ATLAS Collaboration], ATL-PHYS-PUB-2014-010 